Int. J. Biol. Chem. Sci. 11(4): 1727-1740, August 2017

ISSN 1997-342X (Online), ISSN 1991-8631 (Print)

Original Paper http://ajol.info/index.php/ijbcs http://indexmedicus.afro.who.int

\title{
Consommation de l'eau en sachet et ses effets socio-environnementaux dans la Commune de Parakou
}

\author{
Offin Lié Rufin AKIYO
}

Département de Sociologie-Anthropologie, FLASH, Université de Parakou, République du Bénin. E-mail: rufinakiyo@yahoo.fr; Tel : (+229)97476110/94207086

\section{RESUME}

L'eau en sachet est devenue un produit de grande consommation dans les grandes communes du Bénin comme Parakou. Mais force est de constater que cette ressource alimentaire livrée aux populations de la Commune de Parakou suscite depuis quelques années des inquiétudes. En effet, plusieurs risques liés à l'hygiène, depuis la production jusqu'à la commercialisation, ne garantissant pas toujours la qualité d'une eau potable seraient à la base de cette situation. L'objectif de cette étude est de mettre en exergue les impacts socioenvironnementaux de la consommation de l'eau en sachets par les populations de Parakou au nord du Bénin. Dans ce cadre, la revue documentaire, l'entretien et l'observation sont les techniques utilisées pour collecter les données, à l'aide d'outils tels que : la grille d'observation, le questionnaire et le guide d'entretien avec 372 enquêtés comme échantillon choisi par grappe. Pour mieux comprendre les conséquences de la consommation de cette eau sur les populations et l'environnement de ce milieu d'étude, le travail de recherche s'est basé sur une méthode quantitative appuyée des données qualitatives. Les résultats de cette étude montrent que la consommation de l'eau en sachet dans cette localité présente non seulement des avantages mais également des inconvénients. La question de la qualité reste et demeure l'un des grands problèmes que suscite la commercialisation de ce produit. Pour ce faire, un accent mérite d'être mis sur la réglementation du secteur de production et de conditionnement des eaux en sachet à travers l'agrément aux structures de production, le respect strict et effectif des différentes consignes de sécurité et d'hygiène, la formation du personnel et des chefs d'unité de fabrication sur l'assurance qualité, l'instauration des sachets biodégradables, le contrôle et le suivi régulier des unités de production par les structures assermentées de l'Etat.

(C) 2017 International Formulae Group. All rights reserved.

Mots clés : Parakou, eau en sachet, avantages, inconvénients, solution.

\section{Consumption of water in sachets and its socio-environmental effects in the Parakou Commune}

\begin{abstract}
The water in pouches has become a product of great consumption in the large municipalities of Benin like Parakou. But it is clear that this food resource delivered to the populations of the Commune of Parakou has raised concerns in recent years. Several hygiene-related risks, from production to marketing, which do not always guarantee the quality of drinking water, would be the basis of this situation. The objective of this study is to highlight the socio-environmental impacts of the consumption of water in sachets by the populations of
\end{abstract}


Parakou in northern Benin. In this context, the literature review, maintenance and observation are the techniques used to collect data, using tools such as: the observation grid, the questionnaire and the interview guide with 372 respondents as a sample selected by cluster. To better understand the consequences of the consumption of this water on the populations and the environment of this study area, the research was based on a quantitative method supported by qualitative data. The results of this study show that the consumption of water in sachets in this locality has not only advantages but also disadvantages. The issue of quality remains one of the major problems with the marketing of this product. To this end, an emphasis should be placed on regulating the production and packaging water sector in sachets through the approval for production structures, strict and effective compliance with the various safety and hygiene regulations, The training of personnel and heads of manufacturing units on quality assurance, the introduction of biodegradable bags, the control and regular monitoring of production units by the sworn state structures.

(C) 2017 International Formulae Group. All rights reserved.

Keywords: Parakou, water in packet, advantages, disadvantages, solution.

\section{INTRODUCTION}

L'eau est la ressource naturelle la mieux partagée dans le monde. En effet, l'homme peut rester plusieurs jours sans manger, mais il ne saurait passer plus de trois jours sans boire de l'eau (Larbi Bouguerra, 2003). De plus, les aliments consommés peuvent être variés ou refusés pour certains, mais on ne peut, en aucun cas, refuser de boire de l'eau car elle est la composante essentielle de tous les produits de consommation (Baron, 2005). Cette ressource vitale présente plusieurs fonctions socioéconomiques et environnementales qu'on ne saurait occulter pour une meilleure gestion des différentes espèces sur la terre (ABE, 1999). La consommation d'une eau de mauvaise qualité constituerait alors une atteinte à la vie de ces espèces. C'est d'ailleurs pour cette raison que la qualité de l'eau de boisson apparaît partout dans le monde comme une question préoccupante (Blé et al., 2009). Cette analyse est aussi partagée par plusieurs acteurs du monde de la recherche comme le Dr. LEE Jong-wook, ex-Directeur Général de l'OMS lors du lancement de la Décennie Internationale d'Action à travers le sujet « L'eau, source de vie » (2005-2015) lorsqu'il montre l'importance de ladite ressource naturelle en ces termes: " L'eau et l'assainissement sont indispensables à la santé publique. Ils en constituent la base, car lorsqu'on aura garanti à tout un chacun, quelles que soient ses conditions de vie, l'accès à une eau salubre et à un assainissement correct, la lutte contre un grand nombre de maladies aura fait un bond énorme ». Au Bénin, on assiste, depuis quelques années, à la commercialisation de l'eau en sachet sous plusieurs appellations dont celle de «Pure water» qui signifie littéralement «eau pure». Dans les villes béninoises en général et à Parakou en particulier, la vente de l'eau en sachet est une activité qui offre plusieurs opportunités socioéconomiques non négligeables aux populations. Mais de plus en plus, la qualité de ce produit emballé ne cesse de susciter de part et d'autres des interrogations pour maintes raisons. Elle n'est souvent pas conforme aux normes de qualité de l'eau potable en République du Bénin. En effet, la loi n'2010-44 du 21 octobre 2010 portant gestion de l'eau en République du Bénin, indique en son article 60 , que «L'eau livrée à la consommation des populations doit être potable. Quel que soit le mode de gestion du service public d'approvisionnement en eau potable, la personne publique compétente veille, sous l'autorité des ministres en charge respectivement de l'eau et de la santé, et conformément aux dispositions du code 
d'hygiène publique, à la qualité de l'eau distribuée et au respect des normes ». Parlant des exigences de la qualité de l'eau de consommation livrée à la population, la loi n ${ }^{\circ}$ 98-030 du 12 février 1999 portant loi-cadre sur l'environnement en République du Bénin stipule également en son article 31 , que « Tout exploitant d'un système public ou privé d'alimentation en eau et l'exploitant d'un établissement public, commercial ou industriel alimenté en eau par une source quelconque d'approvisionnement, qui mettent de l'eau à la disposition du public ou de leurs employés pour des fins de consommation humaine doivent se conformer aux normes en vigueur ». Mais de plus en plus, il se révèle que le souci des producteurs de cette ressource très sollicitée par les populations est de fournir l'eau en quantité sans se soucier de sa qualité (Alassane Mama Chabi, 2015). L'objectif qualitatif et hygiénique est alors relégué au second rang par certains producteurs dans la ville de Parakou. La consommation de l'eau en sachet insalubre expose les populations à plusieurs risques notamment les maladies hydriques. De plus, une fois l'eau consommée, il se pose le problème de gestion des emballages. En effet, jetés après consommation dans la nature, ces emballages obsolètes, sous l'action du vent et des eaux de ruissellement, sont disséminés presque partout, rendant ainsi la ville de Parakou malpropre. Ce sont-là autant de mobiles qui justifient le présent sujet de recherche.

\section{MATERIEL ET METHODES}

\section{Présentation du milieu d'étude}

Limitée au Nord par la Commune de N'Dali, au Sud, à l'Est et l'Ouest par la Commune de Tchaourou, la Commune de Parakou (Figure 1) s'étend sur une superficie de $441 \mathrm{~km}^{2}$ dont $66 \%$ est urbanisée (PDC de PARAKOU, 2014). Elle est située à $407 \mathrm{~km}$ de la capitale économique Cotonou et représente la principale ville du Nord-Bénin. Avec une altitude moyenne de $350 \mathrm{~m}$, elle est localisée entre $9^{\circ} 15^{\prime}$ et $9^{\circ} 30$ de latitude Nord et entre $2^{\circ} 20^{\prime}$ et $2^{\circ} 45^{\prime}$ de longitude Est. Ce milieu d'étude est subdivisé en trois (03) arrondissements que sont: le $1^{\text {er }}$ Arrondissement, le $2^{\text {ème }}$ Arrondissement et le $3^{\text {ème }}$ Arrondissement.

Avec une population de 255478 habitants en 2013 (Projection du RGPH4/INSAE, 2013), une superficie $441 \mathrm{~km}^{2}$ et une densité moyenne de 510 habitants $/ \mathrm{km}^{2}$, ce milieu d'étude dispose d'un relief à vallons marqué par une succession de croupes de sommet arrondi. Les pentes faibles et peu accidentées sont comprises entre $1,5 \%$ et $4 \%$. La Commune de Parakou bénéficie également d'un climat tropical humide de type soudanien caractérisé par une saison pluvieuse qui s'étend de mai à octobre et une saison sèche allant de novembre à avril. Avec un contexte orographique favorable, les précipitations ont une hauteur moyenne annuelle de $1200 \mathrm{~mm}$ avec un maximum survenant entre juillet, août et septembre. C'est avec l'harmattan qu'on enregistre les plus basses températures dans les mois de décembre et de janvier. Cette commune est une zone partagée par le bassin de l'Okpara et le bassin de Yéroumarou. Les marigots et les ruisseaux de cours temporaire constituent l'essentiel de son réseau hydrographique. Les sols sont globalement à texture légère, d'épaisseur importante, faiblement érodés, mais caractérisés par un fort lessivage des minéraux ; ce qui réduit leur fertilité. Les bas-fonds sont des prairies marécageuses de savanes, des buissons de bambous (Bambusa arundinacca). La croissance de ce milieu d'étude a engendré la transformation des espaces maraîchers en rues collectrices d'eau et zones constructibles.

La situation administrative de la Commune de Parakou est présentée dans la Figure 1. 


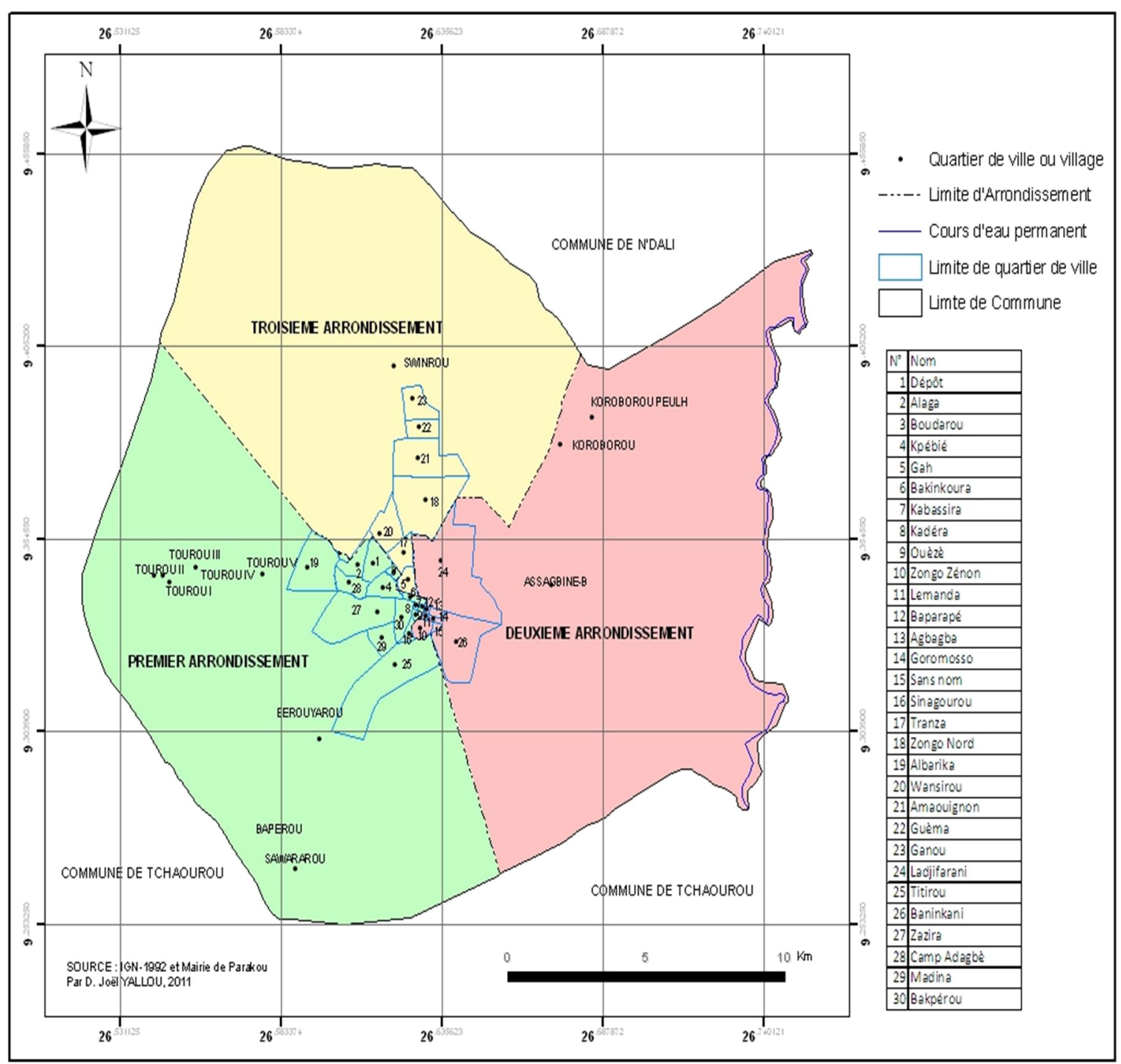

Figure 1 : Carte administrative de la Commune de Parakou. Source: IGN 1992 et Mairie de Parakou.

\section{Méthode}

Dans le cadre de ce travail, une carte sommaire de reconnaissance du cadre d'étude, des fonds de cartes tirés de la carte générale du Bénin IGN mise à jour en 1992 et de l'Atlas monographique du Borgou, une moto marque SANILI, un appareil photo numérique pour les prises de vue, les images satellitaires LandSat ETM janvier 1996- Novembre 2008 et un appareil Global Positionning System (GPS) ont été utilisés.
L'approche méthodologique adoptée comporte trois phases: la collecte des données, le traitement et l'analyse des résultats grâce aux techniques, outils et méthodes appropriés. Outre la documentation, la collecte des données sur le terrain a été effectuée à l'aide des outils tels que le questionnaire, le guide d'entretien et la grille d'observation. Le critère fondamental $\mathrm{du}$ choix des personnes est d'avoir au moins 
consommé une fois l'eau en sachet. Ce critère a permis de mieux apprécier les implications socioéconomiques et environnementales du produit au niveau des principaux acteurs que sont les consommateurs. Les personnes enquêtées au niveau des importants pôles de consommation sont réparties comme suit dans le Tableau 1.

En dehors de ces acteurs privilégiés, un accent est aussi mis sur huit (08) autres catégories d'informateurs en fonction de leur niveau de connaissances sur les maladies hydriques et la pollution de l'environnement causée par les emballages. Il s'agit, notamment de: agents de santé (03), de la police sanitaire (03), d'hygiène et d'assainissement de base (03), de la Direction de l'Alimentation et de Nutrition Appliquée (03), de la Direction Départementale de l'Environnement Borgou/Alibori (03), du Service Départemental de l'Eau Borgou/Alibori (03), les producteurs d'eau en sachet, les ONGs intervenant dans les secteurs de l'eau (03), de l'environnement (03) et l'Association des consommateurs de Parakou (03). Quant à l'observation directe effectuée à l'aide d'une grille d'observation, elle a été réalisée au niveau de 12 consommateurs et de
10 producteurs qui n'ont pas été pris en compte au niveau des enquêtes pour mieux comparer les informations en vue d'une meilleure triangulation.

Au total, dans le cadre de la collecte des informations, les consommateurs de l'eau en sachet des lieux de culte et de cérémonies ont été soumis au guide d'entretien tandis que ceux des milieux éducatifs et d'activités sportives ont eu droit à des questionnaires.

\section{Analyse des données}

Le traitement des données collectées a été effectué suivant cinq étapes: la vérification des fiches de collecte des données, le dépouillement et la codification des différentes fiches de collecte des informations, la saisie des données en tableaux, graphiques et cartes à l'aide des logiciels Word et Excel, la fusion et le transfert de la base de données dans "SPSS" pour analyse. L'analyse des résultats a été faite à l'aide du modèle SWOT pour faire ressortir les forces, les faiblesses, les menaces et les opportunités liées à la consommation de l'eau en sachet dans ce milieu d'étude.

Tableau 1 : Sites de grande consommation de l'eau en sachet à Parakou.

\begin{tabular}{l|c}
\hline Milieu & Effectif des enquêtes \\
\hline Lieux de culte (mosquées, églises, couvents) & 20 \\
\hline Milieux éducatifs (écoles, collèges, universités) & 274 \\
\hline $\begin{array}{l}\text { Lieux de cérémonies (mariage, baptême, décès, séminaires, } \\
\text { conférences, etc.) }\end{array}$ & 48 \\
\hline $\begin{array}{l}\text { Lieux d'activités sportives (stade, terrain de foot, terrain de } \\
\text { sport) }\end{array}$ & 30 \\
\hline TOTAL & $\mathbf{3 7 2}$ \\
\hline
\end{tabular}

Source : Enquêtes de terrain, décembre 2015 


\section{RESULTATS}

Les conditions de production et de commercialisation de l'eau en sachet

Plusieurs indicateurs permettent

d'apprécier les conditions de production et de commercialisation de l'eau en sachet dans la Commune de Parakou. Il s'agit notamment des sources d'approvisionnement, du processus d'emballage, de conservation et du transport, etc.

L'approvisionnement de la matière première qu'est l'eau pour la production de l'eau en sachet varie d'un producteur à un autre. Les investigations en milieu d'étude révèlent que la majorité des producteurs ont recours à l'eau de la Société Nationale des Eaux du Bénin (SONEB) qui, à priori est potable. Mais certaines recherches notamment celles de Otchoumaré (2014) et Allassane Mama Chabi (2015) remettent en cause la potabilité de cette eau en raison des dépôts noirâtres qu'elles contiennent et du fait de certaines contraintes environnementales comme le comblement et l'ensablement de la cuvette du barrage de l'Okpara, d'où elle est prélevée (Otchoumaré, 2014). En dehors de la SONEB, l'eau en sachet est également produite à partir d'autres sources très peu fiables présentées dans le Tableau 2.

$\mathrm{Au}$ regard de ces données du Tableau 2, il ressort que c'est l'eau de la SONEB qui est dominante $(72,8 \%)$ en matière de source d'approvisionnement, vient ensuite, celle de forage $(12,8 \%)$. L'avantage de l'eau de la SONEB par rapport aux autres sources est qu'elle subit des traitements physicochimiques avant sa mise en consommation même si, certaines inquiétudes ne sont toujours pas dissipées concernant sa qualité.

En ce qui concerne les conditions de manipulation du produit, il convient de noter qu'elles se font sans aucun soin au niveau des différents sites sillonnés. Les ouvriers font le travail sans utilisation des Equipements de Protection Individuelle (EPI) comme les gants, les bottes, les lunettes, les casques, les blouses, etc. L'absence du port de gants et de vêtement de protection-type laboratoire constitue une grande inquiétude pour la qualité du produit commercialisé et à la sécurité des travailleurs. La plupart des sites de production ne disposent non plus de réservoirs contenant de l'eau à conditionner. Quant aux filtres, ils sont parfois sales et leur nombre varie de deux à trois. Le nombre de travailleurs dont le niveau d'étude le plus élevé est la classe de $3^{\text {ème }}$ varie en fonction de la demande. L'ensachage se fait par les femmes et un technicien pour la réparation de la machine en cas de panne. D'après certains promoteurs, il suffit d'avoir la volonté et quelques notions d'hygiène pour travailler au niveau de l'entreprise. Cet état de choses se justifie à travers les propos du promoteur $\mathrm{X}$ en ces termes : "Pour travailler dans mon entreprise, on n'a pas besoin d'avoir un gros diplôme, mais il suffit d'aimer la propreté et le travail ». Cette situation relance une fois encore le débat sur le rapport entre le niveau d'étude de l'agent de production et la qualité $\mathrm{du}$ produit obtenu quand on sait que, cette activité nécessite la maîtrise de certaines règles d'hygiène. La Photo 1 met en exergue une étape du processus de production de l'eau en sachet.

\section{Le processus de transport de l'eau en sachet et les conditions de travail des acteurs}

Un autre volet sur lequel l'accent est mis pour mieux apprécier les conditions de production et de commercialisation de l'eau en sachet, est le transport. A cette étape, il faut noter qu'après production, l'eau en sachet est distribuée à des revendeurs par le biais des tricycles et des camions dont l'état des moteurs reste encore à désirer. Les conducteurs de ces engins qui perçoivent des salaires dérisoires, sont sans sécurité sociale et font chaque jour le tour des clients pour livrer le produit. En effet, chaque producteur fixe le salaire de ses agents en fonction de la quantité d'eau vendue et selon les périodes de vente en bafouant royalement le code du travail. De plus, ils sont à la fois chauffeurs et manœuvres pour décharger les produits au 
niveau des différents sites de vente; ce qui les fatigue énormément. Cette situation socioéconomique des conducteurs à l'instar des autres agents de la chaîne de production n'augure pas d'une bonne condition d'hygiène accordée au transport de cette ressource vitale. La preuve, c'est que les produits sont transportés sur des kilomètres depuis l'usine de production vers les centres de vente sans aucune mesure de protection comme les bâches (Photo 2) pour limiter les effets de la poussière, les éventuelles intempéries du soleil et de la pluie, etc. qui peuvent affecter la qualité sanitaire de l'eau comme la perforation des emballages, la destruction progressive de certains nutriments et autres sels minéraux contenus dans l'eau et la libération dans l'eau des éléments nocifs comme des perturbateurs endocriniens, du Bisphénol A, de l'antimoine, etc. qui détruisent le goût de l'eau (Abawi et Widmer, 2010).

La photo 2 montre un camion sans mesure de protection chargé de l'eau en sachet. Tout ce processus mis en place sur les plans humain et matériel n'est pas sans conséquences non seulement sur les populations mais également sur leur milieu de vie.

Tableau 2 : Sources d'approvisionnement de l'eau en sachet.

\begin{tabular}{l|c|c|c|c}
\hline Dénomination & Effectifs & Pourcentage & Pourcentage valide & Pourcentage cumulé \\
\hline Puits & 38 & 10,2 & 10,2 & 10,2 \\
\hline SONEB & 271 & 72,8 & 72,8 & 83,1 \\
\hline Forage & 47 & 12,6 & 12,6 & 95,7 \\
\hline Fontaine & 8 & 2,2 & 2,2 & 97,8 \\
\hline Autres & 8 & 2,2 & 2,2 & 100,0 \\
\hline Total & 372 & 100,0 & 100,0 & \\
\hline
\end{tabular}

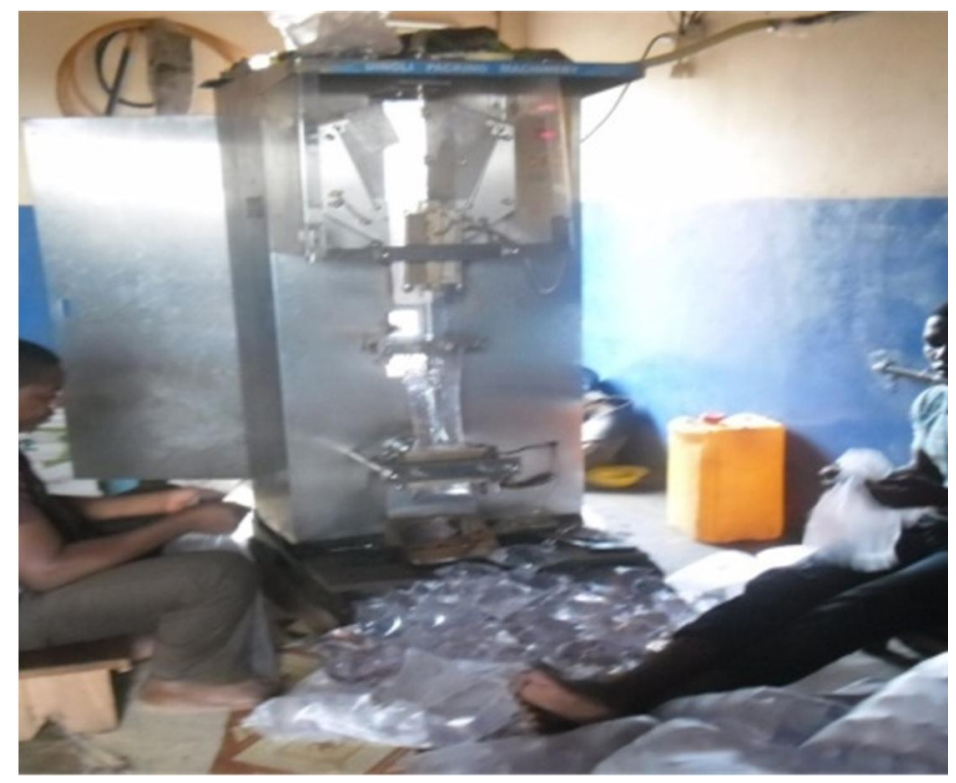

Photo 1 : Mise en sachets de l'eau sur un site de production à Parakou. 


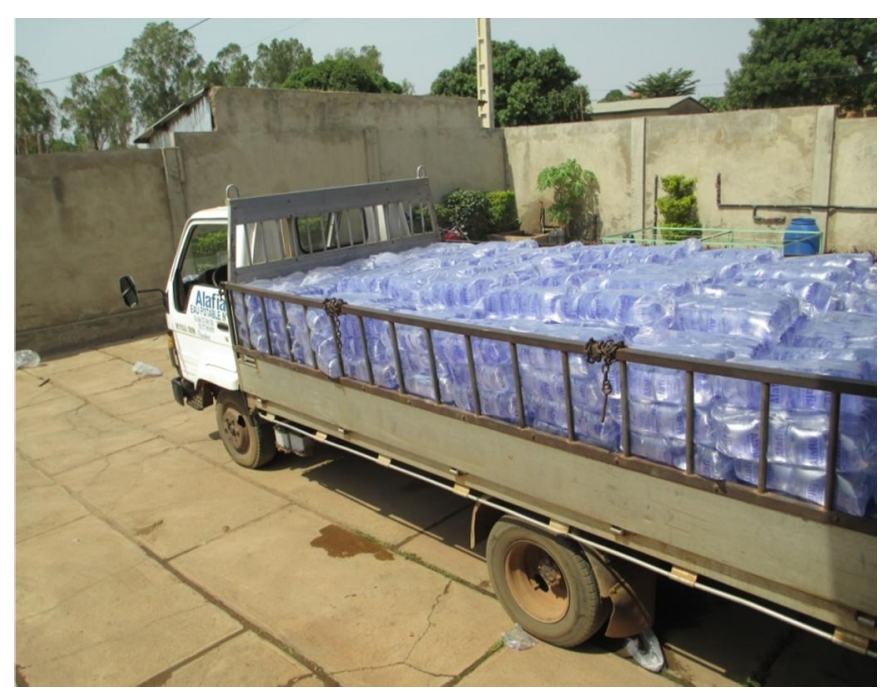

Photo 2 : Transport de l'eau en sachets sans mesure de protection adéquate d'un site de production de Parakou vers les vendeurs.

\section{DISCUSSION}

La commercialisation de l'eau en sachet procure non seulement des avantages mais également des inconvénients qu'il importe d'aborder au niveau de ce milieu d'étude afin d'apporter des mesures correctives en cas de nécessité.

\section{Effets socioéconomiques de la} consommation de l'eau en sachet

A Parakou comme dans bon nombre de villes du Bénin, plusieurs opérateurs économiques interviennent dans la commercialisation de l'eau en sachet pour subvenir aux besoins des consommateurs en eau d'une part, et accroître aussi leurs chiffres d'affaires d'autre part. Elle constitue alors une source d'autonomisation financière pour les opérateurs économiques exerçant dans ce secteur (Jaglin, 2001). En effet, avec $4 \mathrm{~m}^{3}$ d'eau de la SONEB à raison de 98 FCFA par $\mathrm{m}^{3}$, ces derniers produisent au moins 8000 sachets d'eau de $500 \mathrm{ml}$ qu'ils revendent par la suite aux populations à un prix forfaitaire de 25 FCFA l'unité soit donc un total de 198000 FCFA avec un bénéfice de 75.000 FCFA ; ce qui n'est donc pas négligeable en matière commerciale. De plus, cette activité génère aussi plus de 1300 emplois aux enfants, jeunes et vieux désœuvrés du milieu d'étude (Otchoumaré, 2014). "C'est une activité à revenu non négligeable permettant à certaines entreprises de créer de l'emploi et d'en tirer grand profit " a déclaré $\mathrm{J}$ un consommateur du produit. La disponibilité de ce produit permet aux populations de satisfaire en tout temps et en tout lieu leur soif et met en relief leur égalité d'accès à la ressource. L'eau en sachet permet aux populations démunies de se satisfaire aussi en boisson sans se rendre dans les buvettes, les bars, les restaurants, etc. C'est dans cette optique que $\mathrm{Z}$, un fidèle consommateur du quartier Titirou déclare : "L'eau en sachet est la boisson du pauvre car elle est disponible partout: sur la voie, au marché, dans les rues, au niveau des feux, au village, etc et à un prix acceptable de 25FCFA; ce qui n'est pas le cas pour l'eau de Possotomè, de Fifa et autres dont les prix varient entre 400 et 500 $F C F A$ ». Cette eau en sachet est préférée par les consommateurs dans un milieu d'étude dont les conditions climatiques ont assez de similitude avec les zones sahéliennes pendant la saison sèche et de sa disponibilité partout et à tout moment. C'est ce que $\mathrm{K}$. soutient par 
ses propos lorsqu'il dit : "Les consommateurs ont accès à l'eau fraîche surtout en période de chaleur. L'eau en sachet assure la garantie de cette ressource pendant les coupures répétées d'eau de la SONEB surtout lors des délestages ». L'eau en sachet répond alors aux exigences d'accessibilité et de disponibilité permettant aux consommateurs d'étancher leur soif en un temps record et de satisfaire d'autres besoins ménagers (Diagne, 2008). Ce produit permet également de satisfaire un nombre important de consommateurs $(87,6 \%)$ surtout lors des évènements heureux ou malheureux comme le baptême, le mariage, les cérémonies funéraires et autres manifestations populaires. L'importance accordée par les populations de Parakou à ce produit justifie la multiplication des entreprises de production dans le milieu d'étude. C'est un marché hautement concurrentiel dominé par des producteurs comme Pure Water, Alafia, Afaf eau potable, Anfanni eau potable, la Vie, Royal eau potable, Sogodo, Adjos Water, Horeb eau potable, Finesse, etc. Les moments de fortes productions sont souvent les mois de février et d'avril où la chaleur est torride avec de fortes demandes de consommation d'eau par les populations. La disponibilité de cette ressource leur permet aussi de satisfaire d'autres besoins alimentaires et religieux comme la préparation des mets, l'ablution, etc.

Mais de plus en plus, la consommation de cette ressource vitale suscite quelques inquiétudes tant au niveau de la population que de certains acteurs de la société civile pour maintes raisons. En effet, l'eau en sachet est produite en majorité $(72,8 \%)$ au niveau des unités du milieu d'étude à partir de l'eau de la SONEB provenant du barrage d'Okpara et le reste $(17,20 \%)$ à partir d'autres sources (puits, forage, fontaine, etc.) dont la qualité demeure toujours douteuse car elle peut être polluée en amont par les pesticides, nitrates et autres polluants (ABE/MEHU, 1998). Même si la ressource de la SONEB paraît potable, certains résultats relatifs à sa qualité posent problème depuis quelques années compte tenu des conditions socio- environnementales du barrage (Otchoumaré, 2014).

$\mathrm{Au}$ regard de toutes ces implications, le conditionnement de l'eau en sachet serait à l'origine de plusieurs pathologies hydriques comme le choléra, les maux de ventre, la diarrhée et la fièvre typhoïde, les infections selon certains consommateurs et acteurs du milieu médical tels que les médecins, les infirmiers et les agents d'hygiène du milieu d'étude. Elle constituerait alors un véritable problème de santé publique en attendant que des résultats de laboratoire viennent le confirmer au niveau des études postérieures.

La Figure 2 présente l'évolution des pathologies relatives à la consommation de l'eau en sachets dans la Commune de Parakou.

De l'observation de la Figure 2, il ressort que la dominance de la pathologie relative à la consommation de l'eau en sachet est le choléra suivi des maux de ventre et le paludisme vient en dernière position. Notons enfin que le traitement de ces maladies d'origine hydrique constituerait en retour une source de dépenses non négligeables pour les populations de la Commune de Parakou dont les revenus s'amenuisent de façon drastique depuis quelques années avec la chute des activités commerciales et la baisse de la pluviométrie due aux actions anthropiques et aux effets néfastes des changements climatiques (Marh, 2008).

De plus, l'emballage plastique dans lequel se retrouve l'eau de consommation libère selon certaines recherches des perturbateurs endocriniens dans cette ressource minérale (Pote, 2013). De ce fait, il pourrait y avoir des effets sur la santé, y compris à faibles doses. Selon une étude conduite par deux chercheurs allemands publiée dans la revue Environmental Science and Pollution Research, l'eau des sachets contient deux fois plus d'hormones (féminines ou masculines) que celle stockée dans des bouteilles en verre ou l'eau du robinet. Autrement dit, le sachet libère dans l'eau ce 
que l'on appelle des perturbateurs endocriniens. Leurs effets à très faible dose sont encore très mal connus, mais ils sont soupçonnés de modifier chez l'homme le développement et les fonctions sexuelles et reproductrices. Martin Wagner et Jörg Oehlman de l'Université Goethe, basée à Francfort, ont également sélectionné vingt emballages en plastique d'eaux minérales vendues en Allemagne et le constat révèle qu'ils contiennent du Polyéthylène Téréphthalate (PET). Ce produit chimique dangereux est également présent dans les bouteilles en plastique de boissons gazeuses, d'huile de cuisine et il tapisse aussi l'aluminium recouvrant l'intérieur des briques de boissons de plusieurs marques. Le PET assure à la fois «la transparence du plastique, sa résistance aux chocs, son faible poids et son imperméabilité à l'eau, aux gaz et aux arômes» d'après ces deux chercheurs.

Enfin, pour mettre en évidence l'activité hormonale du plastique, Martin Wagner et Jörg Oehlman ont utilisé des levures génétiquement modifiées et des petits escargots d'eau douce, deux modèles de laboratoire classiques pour ce type d'étude. Les levures réalisées à partir des OGM (Organismes Génétiquement Modifiés) ont la particularité de changer de couleur en présence d'infimes quantités d'hormones. Chez les petits mollusques, c'est leur reproduction et leur multiplication qui peuvent varier considérablement en fonction des hormones présentes dans l'eau. Le comportement des levures et des mollusques après un long séjour de près de deux mois dans l'eau n'est pas le même dans les vingt bouteilles en plastique de différents types et les bouteilles témoin en verre. Dans douze d'entre elles, ces chercheurs ont noté dans l'eau des bouteilles en plastique une activité hormonale deux fois plus élevée sur les levures. Les mollusques, qui se reproduisent par parthénogenèse (sans mâle), ont eu deux fois plus de petits escargots dans les bouteilles en plastiques à cause des hormones féminines. Ils ont noté aussi deux choses intéressantes : l'eau ayant séjourné dans les briques est plus polluée par ces perturbateurs. En revanche, l'eau des bouteilles en plastique réutilisables conçues pour être plongées dans l'eau bouillante pour être désinfectées, pratique courante en Allemagne-est moins polluée que les autres dès la deuxième utilisation.

Le Tableau 3 présente l'évolution des cas de prise en charge sanitaire après consommation de l'eau en sachet.

Il ressort de ce tableau que $53,2 \%$ des enquêtés reconnaissent que la consommation de l'eau en sachet entraîne des pathologies ayant des coûts de traitement et 43,2\% mettent l'accent sur le coût lié à l'hospitalisation. Au total, il se révèle que lorsque l'eau est mal traitée ou n'est pas potable, les consommateurs peuvent souffrir des maladies dont les frais de traitement ne sont souvent pas négligeables et impactent négativement sur l'amélioration de leurs conditions de vie (Biaou, 2000).

En dehors du domaine socioéconomique, quelle est l'influence de la consommation de l'eau en sachet sur l'environnement?

Effets environnementaux de la consommation de l'eau en sachet

L'eau en sachet une fois consommée, les emballages sont jetés un peu partout dans la Commune de Parakou sans aucune mesure de protection et recouvrent le sol. Ce commerce constitue alors une source d'insalubrité dans ce milieu d'étude.

Le Tableau 4 présente les différentes utilisations faites des emballages après consommation de la ressource

Ce tableau révèle une mauvaise gestion des sachets d'eau après usage car $66,9 \%$ des enquêtés ont l'habitude de les jeter par terre et $29,6 \%$ dans des poubelles. Cette gestion faite des sachets après usage du contenu contribue à la pollution de la Commune de Parakou. Notons aussi que les consommateurs pour la plupart, sont conscients de la mauvaise gestion de ces emballages vides d'eau mais n'ont pas une solution appropriée. En ce sens, 
Z. affirme ce qui suit: "Je sais bien comme beaucoup de citoyens de mon quartier, que les sachets plastiques polluent l'environnement mais on ne sait où les mettre ».

En effet, ces sachets biodégradables une fois déversés dans la nature sont emportés par le vent et la pluie par système de lessivage vers les différents écosystèmes (sols, eaux, etc.) pour se retrouver plus tard dans la chaîne alimentaire par infiltration où ils libèrent par la suite des émanations et substances toxiques pour l'homme, les plantes et les animaux (Fangnon, 2012). Ces sachets plastiques produits à base du pétrole et plusieurs autres composants chimiques lorsqu'ils sont mal gérés contribuent aussi à l'obstruction des marécages, des caniveaux avec pour corollaire, la difficulté d'écoulement des eaux de ruissellement et la destruction de certaines infrastructures routières.

De plus, certains usagers de l'eau en sachets des quartiers de Wansirou, Kpéro Guéra et Ganon brûlent par intermittence les sachets plastiques après consommation de la ressource par ignorance des normes environnementales régissant la gestion de ces produits dangereux. Ces emballages vides gérés sous cette forme dégagent dans l'environnement, certaines substances toxiques comme le gaz carbonique, le trioxide d'antimoine, le chlorure de polyvinyle, etc. qui polluent en retour l'atmosphère et contribuent par ricochet à l'effet de serre avec pour conséquences, la dégradation de la couche d'ozone et le développement des effets néfastes des changements climatiques (ABE/MEHU, 1998 ; Akakpo, 2012). C'est pour montrer la gravité de la mauvaise gestion des emballages dudit produit qu'un agent de la Police Environnementale du Borgou/Alibori affirme : "Un sachet jeté par terre fait100 à 400 ans avant d'être détruit et cela constitue des ennuis sanitaires et environnementaux. C'est d'ailleurs pour cette raison, que plusieurs maladies sans justification réelle sont au rendez-vous aujourd'hui ».

La photo 3 montre une facette de la mauvaise gestion des sachets plastiques après consommation de l'eau dans le milieu d'étude.

Notons également que la mauvaise gestion de ces sachets est due à plusieurs raisons comme l'incivisme des consommateurs, l'ignorance des conséquences par certains consommateurs, l'absence de poubelles sur les grandes artères et la méconnaissance des textes fondamentaux régissant la protection de l'environnement aux plans international et national.

Ce sont-là autant de résultats de recherche qui suscitent par moments des inquiétudes sur la qualité de l'eau en sachets très commercialisée dans la Commune de Parakou.

Tableau 3 : Coût lié à la prise en charge sanitaire des consommateurs de l'eau en sachet

\begin{tabular}{|c|c|c|c|c|}
\hline $\begin{array}{l}\text { Coût lié aux } \\
\text { soins }\end{array}$ & Effectifs & $\begin{array}{c}\text { Pourcentage } \\
(\%)\end{array}$ & $\begin{array}{l}\text { Pourcentage } \\
\text { valide (\%) }\end{array}$ & $\begin{array}{l}\text { Pourcentage } \\
\text { cumulé (\%) }\end{array}$ \\
\hline $\begin{array}{l}\text { Coût lié à } \\
\text { l'hospitalisation }\end{array}$ & 161 & 43,2 & 43,2 & 43,2 \\
\hline $\begin{array}{l}\text { Coût lié au } \\
\text { traitement }\end{array}$ & 198 & 53,2 & 53,2 & 53,2 \\
\hline Autres à préciser & 13 & 3,5 & 3,5 & 96,5 \\
\hline Total & 372 & 100,0 & 100,0 & \\
\hline
\end{tabular}




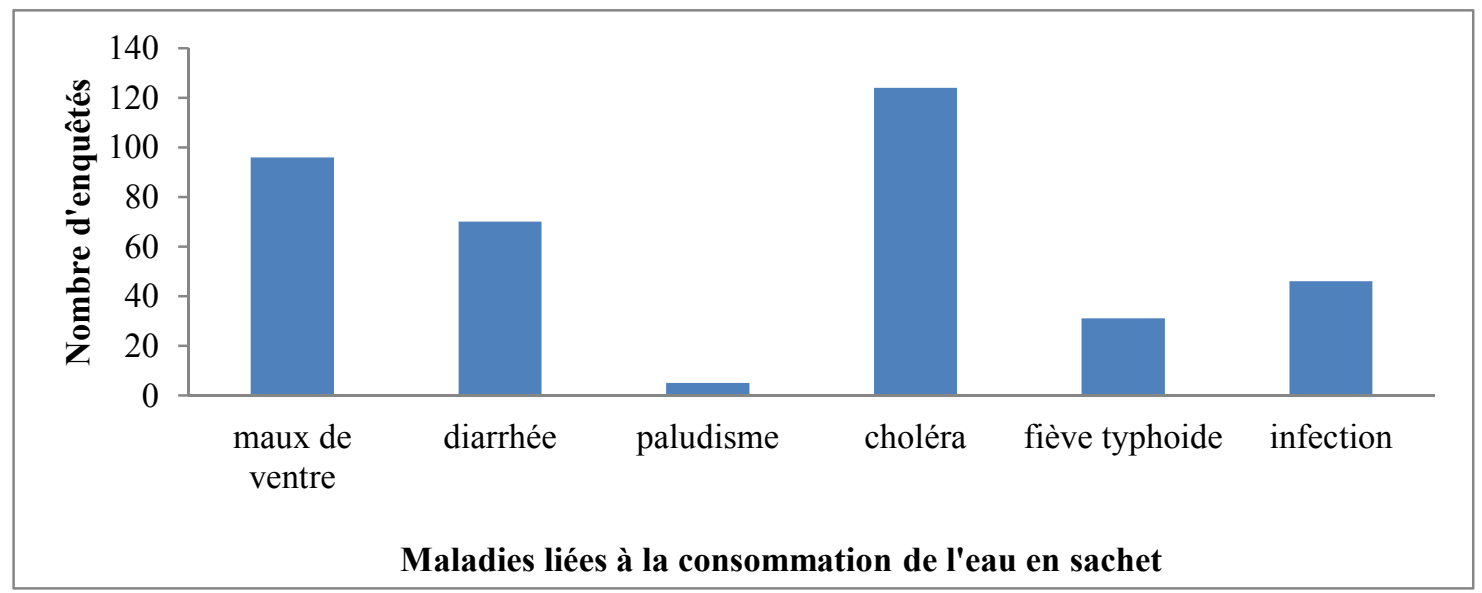

Figure 2: Maladies liées à la consommation de l'eau en sachet à Parakou. Source : Enquêtes de terrain et registres des centres de santé, décembre 2015.

Tableau 4 : Différents usages faits des emballages vides d'eau.

\begin{tabular}{lllll}
\hline Lieu de dépôt & Effectifs & Pourcentage (\%) & Pourcentage valide (\%) & $\begin{array}{l}\text { Pourcentage } \\
\text { cumulé (\%) }\end{array}$ \\
\hline Poubelle & 110 & 29,6 & 29,6 & 29,6 \\
\hline Par terre & 249 & 66,9 & 66,9 & 96,5 \\
\hline Incinération & 2 & 0,53 & 0,53 & 97,0 \\
\hline $\begin{array}{l}\text { Trou } \\
\text { ordures }\end{array}$ & 7 & 1,9 & 1,9 & 98,9 \\
\hline Caniveaux & 2 & 0,53 & 0,53 & 99,5 \\
\hline Autres & 2 & 0,53 & 0,53 & 100,0 \\
\hline Total & 372 & 100,0 & 100,0 & \\
\hline Source : Enquêtes de terrain, décembre 2015 & &
\end{tabular}

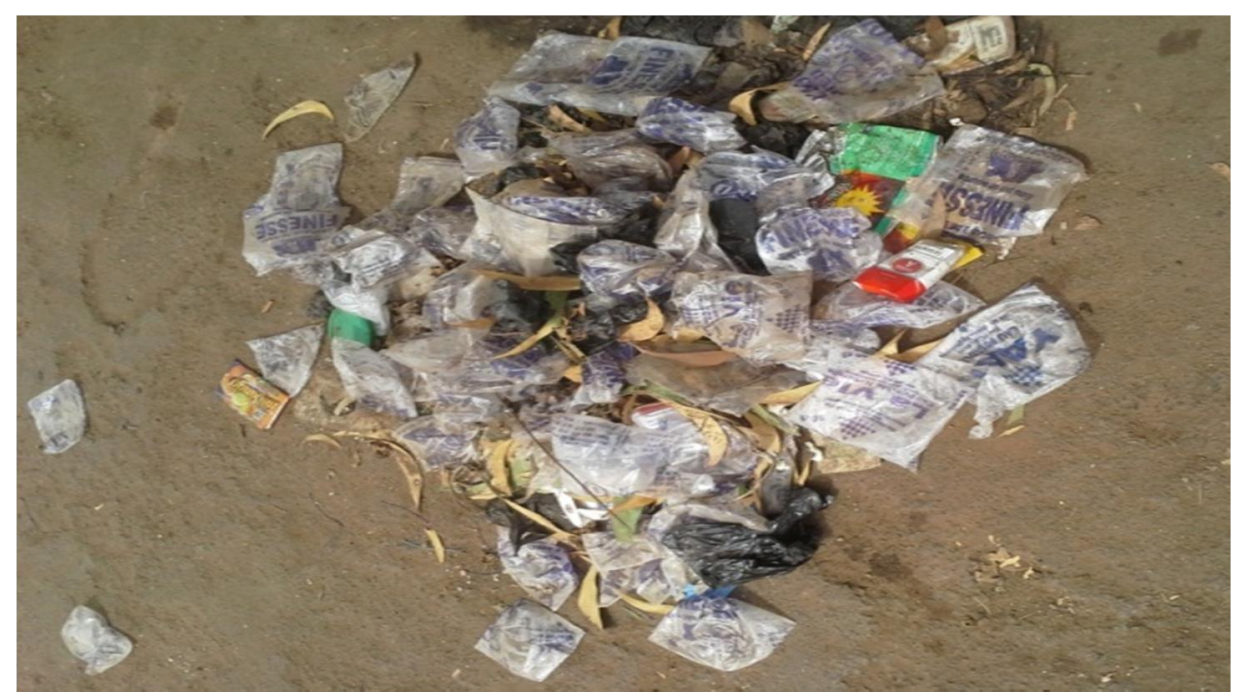

Photo 3 : Les sachets de l'eau «Pure water» jetés dans une ruelle du quartier Oké Dama. Source : Prise de vue, Alassane Mama Chabi, décembre 2015. 


\section{Conclusion}

En définitive, la commercialisation de l'eau en sachets a pris de l'ampleur ces dernières années au Bénin en général et dans la Commune de Parakou en particulier avec la croissance démographique et la pénurie d'eau due aux effets néfastes des changements climatiques. Le développement de cet important marché de l'eau de boisson conditionnée présente non seulement des avantages mais également des inconvénients tant pour l'Etat, les populations que pour l'environnement. En effet, la majeure partie des eaux en sachet est commercialisée de façon illégale dans le milieu d'étude avec des emballages peu indiqués et des conditions d'hygiène peu orthodoxes. Cette situation pose alors le problème d'application des normes environnementales, gage d'une meilleure santé publique. De plus, ce secteur échappe considérablement au contrôle de l'État, faute de textes appropriés dans le domaine ou parce qu'ils sont peu connus ou respectés par les acteurs de la chaîne de production. Dès lors, la question de contrôle et du suivi de la qualité de cette importante denrée alimentaire qui dessert des milliers de citoyens dans la Commune de Parakou s'avère nécessaire pour garantir un environnement sain et une population en bonne santé. $\mathrm{Au}$ regard à tous ces impacts constatés sur les plans social, économique et environnemental dans ce secteur au niveau du milieu d'étude et surtout au regard des sources d'approvisionnement (puits, fontaine, forage), l'Etat est appelé à prendre ses responsabilités pour assurer une sécurité alimentaire et environnementale durable aux populations.

Pour ce faire, il est impérieux d'« agir pour un mieux-être » des consommateurs afin que l'Etat communique davantage avec les populations sur les textes régissant l'environnement en général et le domaine de l'eau en particulier, mette en place ou actualise les textes régissant le contrôle et le suivi du secteur, forme les promoteurs sur la gestion environnementale du produit et exige d'eux un agrément de l'Etat, mette les ressources humaines et matérielles nécessaires à la disposition des services d'hygiène et d'environnement pour un suivi régulier des sites de production et dote enfin, les grandes artères de la Commune en poubelles. C'est à ce prix que le pari du développement durable serait une réalité au Bénin en général et dans ce milieu d'étude en particulier.

\section{REFERENCES}

Abawi GS, Widmer TL. 2010. Impact of soil health management practices on soil borne pathogens, nematodes and root of diseases vegetable crops. Appl. Soil Ecol., 15: 13-47.

ABE. 1999. Loi $n^{\circ} 98-030$ Portant $d u 12$ Février 1999 Portant Loi-Cadre sur l'Environnement en République $d u$ Bénin. Collection $2^{\text {eme }}$ édition les Presses du JORB: Porto-Novo, 66 p.

ABE/MEHU. 1998. Normes et standards environnementaux : principales sources de pollution de l'air et de l'eau au Bénin, Volume 1, 45 p.

Akakpo RH. 2012. Gestion des contraintes et urbanisation à Djidja, Mémoire de Maîtrise de Géographie FLASH/UAC, Abomey-Calavi, $70 \mathrm{p}$.

Alassane Mama Chabi S. 2015. Incidences socioéconomiques et environnementales de la consommation de l'eau en sachet dans la ville de Parakou, Mémoire de Licence professionnelle en SociologieAnthropologie, Option Médiation Sociale et Facilitation de Développement FLASH/UP, Parakou, 80 p.

Assemblée Nationale du Bénin. 2010. Loi n'2010-44 du 21 Octobre 2010 Portant Gestion de l'Eau en République du Bénin. Presses du JORB. Porto-Novo, $23 \mathrm{p}$.

Baron C. 2005. «Société civile et marchandisation de l'eau: Expériences internationales », Sciences de la société, $\mathrm{n}^{\mathrm{o}}$ 64, février, Toulouse, PU Mirail, $249 \mathrm{p}$.

Biaou G. 2000. Coopérer et agir Autrement pour un Mieux-Etre: Stratégie et Action 
du Centre Béninois pour le Développement Durable. Les éditions du Flamboyant : Cotonou, Bénin ; 253 p.

Blé LO, Mahaman BS. 2009. Étude de la Potabilité des Eaux de Boisson Conditionnées en Côte d'Ivoire : Cas des Eaux de la Région du Grand Abidjan. European Journal of Scientific Research, 28(4): 552-558.

Diagne A. 2008. L'accès des ménages pauvres à l'eau potable dans les banlieues de Dakar. In Le Sénégal Face aux Défis de la Pauvreté. Les Oubliés de la Croissance. Daffé G, Diagne A (éds). Éditions Karthala, CRES et CREPOS. Paris ; 51-79p.

Fangnon B. 2012. Qualité des sols, systèmes de production agricole et impacts environnementaux et socioéconomiques dans le département du Couffo au sudouest du Bénin, Thèse de Doctorat Unique, $308 \mathrm{p}$.
Jaglin S. 2001. L'eau potable dans les villes en développement: les modèles marchands face à la pauvreté. TiersMonde, 42(166): 275-303.

Larbi Bouguerra M. 2003. Les Batailles de l'Eau. pour un Bien Commun de l'Humanité, Enjeux Planète : Paris ; 239.

Marh P. 2008. Evolution du secteur agricole et des conditions des ménages au BurkinaFaso. Projet AI/CN-SISA, 92 p.

Otchoumare COM. 2014. Problématique de la gestion du barrage de l'Okpara par la SONEB dans le cadre de l'approvisionnement en eau potable de la ville de Parakou, Mémoire de Licence Professionnelle en SociologieAnthropologie, UP/FLASH, 73 p.

Pote J. 2013. Concentration of metals in surface water and sediment of Luilu and Musonie Rivers, Klowezi-Katanga, Democratic Republic of Congo, Applied Geochemistry, 39: 26-32. 